\title{
GESCHICHTSSCHREIBUNG ALS KONSTRUKTIONSHANDLUNG. Anmerkungen ZU GesChichte Und GegenWART DER MUSIKGESCHICHTSSCHREIBUNG
}

\author{
Helmut Rösing
}

\section{Anfänge der Geschichtsschreibung}

Seit Menschengedenken wurden einschneidende Ereignisse - religiöse Offenbarungen, besondere Naturereignisse, Katastrophen und Kriege - in das kollektive Gedächtnis eingeschrieben und an die nachfolgenden Generationen weitergegeben. Dies geschah mündlich durch die kontinuierliche Überlieferung in der Form von Geschichten, Erzählungen, Epen, es geschah bildnerisch bzw. schriftlich durch die Fixierung auf Stein, Holz, Papier und musikalisch durch epische Gesänge, Lernlieder oder auch Geräuschimitationen (z.B. die Wiedergabe eines Sturms, eines Vulkanausbruchs oder Erdbebens, siehe dazu Graf 1966) in einem Musikstück.

Das macht deutlich: Kulturen ohne Geschichtsbewusstsein gibt es nicht. Allerdings ist ein diesbezüglicher Nachweis bei schriftlosen Kulturen weitaus schwerer zu erbringen als bei den Hochkulturen. Seit ihren ersten Anfängen zwischen Euphrat und Tigris gilt hier die Vergegenwärtigung von Vergangenheit durch wie auch immer geartete Geschichtsschreibung ebenso wie der astrologisch und astronomisch fundierte Blick in die Zukunft als unverzichtbar für die Festlegung und Ausdeutung des jeweils gültigen religiösen, politischen und kulturellen Selbstverständnisses. Grundlegende Ereignisse wurden nicht zuletzt auch aus diesem Grund bildnerisch auf Reliefs, Stelen oder Triumphbögen und schriftlich auf Steinplatten bzw. Papyrusrollen festgehalten.

Das ist der Beginn einer »expliziten Historiographie«. Sie wurde seit dem 3. J ahrtausend v. u.Z. immer mehr verfeinert. Die Anfang des 3. J ahrhunderts v.u.Z. eingerichtete alexandrinische Bibliothek mit weit über 
700.000 Buchrollen legt davon ein beeindruckendes Zeugnis ab. Sie galt bis zu ihrer Zerstörung in spätrömischer Zeit als das wissenschaftliche Zentrum der Antike.

\section{Beginn der expliziten Musikgeschichtsschreibung}

Musikgeschichtsschreibung dagegen ist - anders als die explizite Historiographie - eher eine Errungenschaft der Neuzeit. Ihre Anfänge liegen in der Zeit der Aufklärung. Entscheidender Auslöser ist ein zunehmend historischmusikgeschichtlich ausgeprägtes Interesse. Das zeigt sich gegen Ende des 18. J ahrhunderts mit Beginn des Historismus bei allen Kunstdisziplinen. Umgesetzt wird es z.B. in den epochalen enzyklopädischen Arbeiten von Denis Diderot und Jean Baptiste le Rond d'Alembert sowie dem »Dictionnaire de Musique« von J ean J acques Rousseau.

Vorläufer dieser historisierenden Entwicklung ist die Musikkritik. Als einer der ersten Musikkritiker im modernen Sinn hat sich in Deutschland J ohann Mattheson einen Namen gemacht. Er schuf 1722 ein Periodikum, das sich ausschließlich mit Musik beschäftigte, die »Critica musica«. In diesem J ournal setzte man sich vor allem mit Stil- und Geschmacksfragen auseinander: mit der salten Polyphonie< (Johann Sebastian Bach) und den neueren melodisch orientierten Richtungen (Telemann, Händel).

Um die seinerzeit gegenwärtigen Musikstile zu verstehen und zu legitimieren, wurde der Blick in die Vergangenheit immer wichtiger. So legte Giovanni Battista Martini zwischen 1757 und 1781 die wohl erste explizite Musikgeschichte vor. Sie umfasste drei Bände, kam aber über die Musik der Alten Welt nicht hinaus. Anders nur wenig später Charles Burney. Die vier Bände seiner General History of Music (1776-1791) gründen auf vielfachen Begegnungen mit führenden Musikern und Musiktheoretikern in ganz Europa. Dabei werden - was durchaus >modern< anmutet - auch Tanzmusik, Organisationsformen des Musiklebens, Aspekte des Instrumentenbaus, der Musikerziehung und des Musikjournalismus in seine Geschichtsschreibung mit einbezogen. Quellenkritische Studien dagegen fehlen.

Dieser die Musik der Zeit und ihr aktuelles musikbezogenes Umfeld beschreibende Ansatz von Burney wird von den Vertretern des in Deutschland neu etablierten universitären Fachs Musikgeschichte bzw. Musikwissenschaft nicht mehr weiter verfolgt. Die auf wissenschaftlicher Quellensichtung beruhenden Bücher zur Musikgeschichte von J ohann Nikolaus Forkel (1788), Raphael Georg Kiesewetter (1846) und Hugo Riemann (1901) - um nur 
einige der bedeutendsten zu nennen - bleiben eingegrenzt auf Aspekte von Werk und Biographie. Ihr Ordnungsprinzip ist streng chronologisch. Die Darstellungen folgen der Maxime, Musik werde desto wahrer und erhabener, je mehr sie sich dem 19. J ahrhundert nähert.

Dieses evolutionäre Bild entspricht der Idee der Aufklärung. Es huldigt der Überzeugung, dass es möglich sei, nicht nur kritisch, sondern auch verbessernd in das öffentliche Musikleben eingreifen zu können. Somit ergänzen sich Musikkritik und Musikgeschichtsschreibung bei der Benennung von musikalischen Werten und Wertmaßstäben. Diese werden zusammengefasst in einer musikästhetischen Grundmaxime: Hochstehende Musikstücke sind autonome Werke, die ihren Sinn allein durch die jeweilige individuelle Formung des musikalischen Zusammenhangs besitzen. Damit geben Musikkritik und wissenschaftliche Musikgeschichtsschreibung dem erstarkenden Verlagswesen, dem Musikalienhandel, dem bürgerlichen Konzertwesen und dem gehobenen Publikum eine Richtung vor, in die ernste und ernst zu nehmende Musik sich einzupassen hat.

Im 19. Jahrhundert war Musikwissenschaft ein Synonym für Musikgeschichte. Fragen nach den Grundlagen und Bedingungen musikalischen Handelns und Verhaltens wurden getreu dem ästhetischen Verständnis der Werkautonomie kaum berücksichtigt. Und Musik aus Alltagskulturen und außereuropäischen Ländern fanden allenfalls am Rand Behandlung. Generell unberücksichtigt blieben - so Georg Knepler noch 1997 -

»die konzeptionellen Menschheits- und Allgemeinheitsansprüche. Eine Antwort auf die große Frage nach dem Zusammenhang von Musik- und Menschheitsgeschichte, die die entscheidenden Probleme und Konflikte erst zusammentreten lässt, wird von den meistverbreiteten Werken über Musikgeschichte [...] umgangen« (Knepler 1997: 1312).

Die Erstarkung der Naturwissenschaften und die Formulierung von grundlegenden Einsichten bzw. >Gesetzen< aus den Fachgebieten Mathematik, Physik, Akustik, Physiologie und Wahrnehmungspsychologie in der zweiten Hälfte des 19. Jahrhunderts gaben Anstoß zu naturwissenschaftlicher Forschung auch im Bereich der Musikwissenschaft. Im Jahr 1860 legte Gustav Theodor Fechner sein viel beachtetes Buch Elemente der Psychophysik vor. Hier ging es um eine >Ästhetik von unten<, die Darlegung von Beziehungen zwischen physikalischen Gesetzmäßigkeiten und ästhetischem Erleben von Musik. Drei Jahre später erschien das auch heute noch grundlegende Buch des musikbegeisterten Mediziners, Physiologen und Akustikers Hermann von Helmholtz: Die Lehre von den Tonempfindungen als physiologische Grundlage für die Theorie der Musik (1863). Im Mittelpunkt der Ausführungen 
steht die experimentelle Überprüfung psychoakustischer Phänomene der Musikwahrnehmung.

Vor diesem Hintergrund ist die programmatische Schrift von Guido Adler über »Umfang, Methode und Ziel der Musikwissenschaft « von 1885 zu verstehen. Sie bedeutet eine Neubestimmung des Fachs Musikgeschichte als Musikwissenschaft. Sein Vorschlag, die bislang meist normativ betriebene Musikästhetik durch psychoakustische und psychologische Ergebnisse zu untermauern, darüber hinaus Hilfswissenschaften wie Akustik und Mathematik, Physiologie und Ethnographie in die Untersuchungen über Produktion, Funktion und Wirkungsweisen von Musik mit einzubeziehen, vor allem aber sein Hinweis auf die gesellschaftliche Bedingtheit von Musik lassen Adlers Konzept auch heute noch als Richtung weisend erscheinen. So hielt er es für geboten, die Entstehungsbedingungen und den Zweck von Musik in jede Musikbeschreibung mit einzubeziehen:

»Die ganze Umgebung des Künstlers und des Werkes ist in Erwägung zu ziehen, die physischen, psychischen und allgemein kulturellen Um- und $\mathrm{Zu}$ stände sind in ihren Verschlingungen zu verfolgen. Alle subjektiven und objektiven Entstehungsmomente sind aufzudecken. Alles, was den Schaffenden betrifft, seine Abstammung, seine Disposition, sein Vorgehen, der Schaffensakt, die zeitliche und örtliche Schaffenslage und Bedingtheit. Neben der Individualpsyche ist die Sozialpsyche seiner Umgebung, das Differenzierende und das Gemeinsame darzulegen « (Adler 1919: 36).

Um diesem Anspruch gerecht werden zu können, schlug Adler die Aufteilung in einen historischen und einen systematischen Zweig der Musikwissenschaft vor. Dieser anspruchsvolle Aufgabenkatalog hat im 20. Jahrhundert zu einer hochgradigen Diversifizierung und Spezialisierung des Faches Musikwissenschaft und der Musikgeschichtsschreibung geführt. Die Einengung auf schriftlich fixierte Musik des Abendlandes wurde aufgegeben. Auch die Musik fremder Kulturen, jede Form von Umgangs- und Gebrauchsmusik und vor allem die das 20. Jahrhundert zunehmend prägenden, überwiegend medial verbreiteten Musikrichtungen wie Schlager, Pop, Rock und J azz sind geschichtsfähig und geschichtswürdig geworden.

In der Musikgeschichtsschreibung führen die verschiedenen Musikrichtungen und Musiksphären allerdings immer noch ein Eigenleben. Eine Zusammenschau, die der Realität des Musiklebens gerecht wird, fehlt nach wie vor. Das ist deswegen befremdlich, weil bei den Musikern ebenso wie in den Medien Rundfunk, Fernsehen und Internet diese Zusammenschau von Weltmusik, volkstümlicher Musik, Klassik und Pop, Rock und Jazz mittlerweile gang und gäbe ist. Die Dichotomie zwischen $U$ und $E$ kann im musikbezoge- 
nen Alltag als überwunden betrachtet werden, sie existiert aber noch in den Köpfen so mancher Vertreter des Fachs Musikwissenschaft.

\section{Veränderungen des Musiklebens durch Notation und elektrische Medien}

Der Überblick über die Geschichte der Musikgeschichtsschreibung zeigt, dass explizite Musikgeschichte ohne die Verfügbarkeit von Musik in notenschriftlicher oder medialer Form kaum möglich ist. Bis in das 9. J ahrhundert hinein blieb Musik ausschließlich gegenwärtig und verfügbar, solange sie erklang. Voraussetzung für den Fortbestand eines Liedes oder instrumentalen Musikstücks war ihre mündliche Überlieferung. Das geschah im Hinblick auf einen bestimmten Anlass und eine bestimmte Aufführungssituation. Musik hatte hier Funktionen zu erfüllen, und diese Funktionserfüllung sicherte ihr in den Köpfen der Anwesenden den Fortbestand über die Zeitdauer ihres realen Erklingens hinaus: im Kult, als Ritus, bei Festen, zur Arbeitserleichterung, zur emotionalen Bereicherung, als Erinnerung an kriegerische Vorgänge, eine erfolgreiche J agd und anderes mehr.

Jede erneute Wiedergabe von Musikstücken aus der Erinnerung heraus konnte und sollte in Anpassung an die aktuelle Darbietungssituation musikalische Veränderungen erbringen. Diese gingen dann als neue Variante in die mündliche Überlieferung ein. Die Aufrechterhaltung einer musikalischen Tradition war somit fragil. Und unterbunden wurde sie, wenn ein Musikstück nicht in der Erinnerung gespeichert und von Generation zu Generation weitergegeben wurde. Dann verschwand es aus dem kollektiven Gedächtnis. Musikgeschichte manifestierte sich somit in dem, was an Musik durch kontinuierliche mündliche Überlieferung präsent blieb.

Die Festschreibung von gesprochenem Wort als Text gehört zu den Merkmalen aller Schriftkulturen seit dem Altertum. Die Verschriftlichung von Musik stellt demgegenüber erst eine Errungenschaft der Neuzeit dar. Die Entwicklung der westlichen Notation vollzog sich in mehreren Schritten vom 9. bis 16. J ahrhundert. Sie ist stark abstrahierend und bedarf der Interpretation, da sie nur die kompositorische Essenz, nicht aber sekundäre Komponenten wie Klangfarbe, Tempo, Betonungen, Spieltechniken u.a.m. absolut verbindlich regelt.

Die Notation von Musik bedeutet einen folgenreichen Eingriff in den musikalischen Schaffensprozess und die musikalische Aufführungspraxis. An die Stelle von gedächtnisgestützter Aufführung, Variantenbildung und Improvisation tritt die Komposition. Erst die Notation führte zu komplexer 
musikalischer Strukturbildung, zu rational und nicht mehr intuitiv begründeten Kompositionsregeln und zum Schaffen von immer wieder neuen, originellen, andersgearteten Formen, Stilen und Genres. Mit den Worten von Christian Kaden (1985: 334): Notation ermöglichte »die Entfaltung der Komposition als einer mehrfach reflektierten, von memorialer Tradierung abgehobenen Form musikalischen Produzierens«.

Komponist und interpretierender Musiker brauchen nun nicht mehr identisch zu sein. Die Interpreten werden zu Dienern des Werkes. Es beginnt eine Spezialisierung und Arbeitsteilung, die sich allein schon am Beispiel des Orchesters gut verfolgen lässt. Bis zum Beginn der musikalischen Klassik war es üblich, dass der Komponist als Instrumentalist oder Solist die Aufführung seiner Werke leitete. Diese Einheit ging im 19. Jahrhundert zunehmend verloren. Die Aufgaben von Solist und Dirigent werden nun jeweils speziell ausgebildeten Musikern zugeordnet, und selbst die Spezialisierung von Orchestern zur Aufführung der Musik bestimmter Epochen und Stile schreitet immer weiter voran. Präzision, Perfektion und die Einbeziehung von historischen Aufführungspraktiken gehören heutzutage zu den Selbstverständlichkeiten des traditionellen Konzertwesens.

Nicht allein die Komposition, sondern darüber hinaus auch die Qualität der Aufführung ist immer mehr maßgeblich am Erfolg eines Werkes bei Publikum, Kritikern und Experten beteiligt. Öffentliche Diskussion und Bewertung entscheiden darüber, ob ein Werk in den Kanon des Konzertrepertoires Eingang findet oder nicht. Damit beginnt es den Gesetzen des Marktes, den Maximen von Verwertung und Verwertbarkeit zu unterliegen. Die Gründung bürgerlicher Musikvereine, die Erstarkung des Verlagswesens, der Zulauf zu Gesangvereinen, die gezielte Pflege von Hausmusik, das Klavier als Statussymbol und der musikalische Salon als gehobener Zeitvertreib sind weitere Indizien dafür. Mündliche Überlieferung wird nur noch in Randgebieten des Musiklebens - etwa bei Volksliedern, Tanzmusik oder Gassenhauern - praktiziert. In den Fokus von Musikkritik und Musikwissenschaft gerät die notierte Musik aus der Vergangenheit und dem, was jeweils gerade Gegenwart ist. Sie stellt den Fundus für das dar, was musikgeschichtlich aufgearbeitet wird.

Durch die Erfindung des Tonträgers im ausgehenden 19. J ahrhundert beginnt sich diese Situation zuerst unmerklich, dann aber immer deutlicher zu ändern. Die Aufzeichnung und Wiedergabe von Sprache und Musik markiert den Beginn der medialen Musiktradierung. Die Folgen sind einschneidend für den Umgang mit Musik. Jede real erklingende Musik, die auf einem Speichermedium festgehalten wird, bleibt zur wiederholten Wiedergabe überall dort verfügbar, wo die technischen Voraussetzungen zur Wiedergabe be- 
stehen - gleichgültig, ob sie zuvor notenschriftlich festgehalten, mündlich tradiert, aktuell improvisiert oder synthetisch erzeugt wurde. Ab der Wende zum 20. J ahrhundert fing man an, in Wien, Paris, Berlin usw. Phonogrammarchive als zentrale Sammelstellen für Tonträgermusik einzurichten. Damit wurde nun auch die nicht in Notenform festgehaltene Musik geschichtsfähig (siehe Elste 1998: 653ff.).

Ihre Speicherung und Vermittlung erfolgt über akustische Medien, audiovisuelle Medien und in letzter Zeit zunehmend über Computer und Internet. Der Übergang von der analogen zur digitalen Technik bedeutet hier so etwas wie eine Art Quantensprung. Wurde früher nur nach künstlerischen, dokumentarischen und/ oder kommerziellen Gesichtspunkten ausgewählte Musik auf Tonträger festgehalten, spielt seit der Digitalisierung das Auswahlkriterium nur noch eine untergeordnete Rolle. Nahezu alles, was erklingt, wird auch festgehalten. So werden z.B. im Deutschen Musikarchiv der Deutschen Nationalbibliothek alle in Deutschland erschienenen und erscheinenden Tonträger gesammelt. Das Deutsche Rundfunkarchiv verfügt neben London und Paris über die weltweit größte Phonothek mit Tonaufnahmen aus allen Epochen und Genres. Und schließlich gibt es spätestens seit der J ahrtausendwende immer mehr weltweit vernetzte Online-Musikdatenbanken wie freeDB, MusicBrainz oder Discogs.

Musik, die die nicht der notenschriftlichen Aufzeichnung, sondern allein der Speicherung auf Ton-/ Datentäger ihre Existenz verdankt, bedarf keiner immer wieder neuen Interpretation. Sie ist in ihrer klingenden Erscheinungsform festgeschrieben. Das hat tief greifende Veränderungen für Musikleben und Musikverständnis zur Folge. Von mündlich überlieferter Musik, deren Merkmal eine mit jeder Darbietung einhergehende Variantenbildung ist, wird eine Variante festgehalten und zum >Werk< erklärt. Anders gesagt: Das analoge bzw. digitale Abbild wird von allen funktionalen Attributen, die Bestandteil des Beziehungssystems Musik sind, bereinigt. Angesichts ihrer medialen Allverfügbarkeit erhält die dem Wesen von erklingender Musik nicht gerecht werdende Fixierung bei beliebig austauschbarer Darbietungssituation den Status der Normalität.

Notierte Musik kann zwar nach wie vor immer wieder in neuen Interpretationen aufgeführt werden, zunehmend aber bieten die aufgezeichneten Produktionen einen Vergleichsmaßstab, dem sich jede neue Interpretationsleistung zu stellen hat. Demzufolge dominiert bei der Darbietung von Werken der musikalischen Klassik heutzutage die Interpretations- gegenüber der Werkkritik. Damit einher geht die Verkrustung des Repertoires. Neue Kompositionen finden kaum noch, in Vergessenheit geratene und wiederentdeckte Werke nur vereinzelt Einlass in den Repertoirekanon. 
Nicht notierte, auf Tonträger präsentierte Musikstücke sind Unikate. Sofern diese Stücke von den gleichen Interpreten wiederholt live aufgeführt werden, unterliegen sie mehr oder weniger dem Gesetz der Variantenbildung. Nehmen sich andere Interpreten des Stücks an, dann wird daraus eine Coverversion. Die Bandbreite der Abweichungen erstreckt sich von einer angestrebten Stückidentität bis hin zu einem neuen Werk. Obwohl somit »der Grad der Veränderung recht unterschiedlich ausfallen kann« wie Marc Pendzich (2004: 2) in seiner umfangreichen Geschichte der Coverversion ausführt -, »bleibt das Originalwerk in seinen wesentlichen Zügen erhalten«.

Neben der Coverversion erhält die Wiederverwertung von Teilstücken medial vorgegebener Musik zunehmende Bedeutung. Digitale Methoden des Sampelns führen zur Ausweitung der herkömmlichen, über die Abstraktionsstufe des musikalischen Notats vollzogenen Zitierpraxis. Digitale Samples von gespeicherter Musik werden als Versatzstücke direkt in neue Musikstücke integriert. Sie tragen somit bereits die Spuren von Geschichtlichkeit in sich. Vieles davon ist allerdings nur für den Experten entschlüsselbar.

Der Wandel von mündlicher über die schriftliche bis hin zur medialen Übertragung von Musik hat somit nachhaltige Konsequenzen für die Musikgeschichtsschreibung. Das betrifft (a) die Fülle des für die Geschichtsschreibung zur Verfügung stehenden musikalischen Materials - sie hat exponential zugenommen -, (b) die Anzahl möglicher Auswahlkriterien, die nun sowohl musikimmanent wie funktional, publikums- bzw. gesellschaftsorientiert, marktbezogen oder technikgeprägt sein können, (c) den Wissenschaftsanspruch, der sich zunehmend auch den Kriterien naturwissenschaftlicher Forschung annähert, (d) die Zielgruppe, die keineswegs ausschließlich das gehobene Bürgertum zu sein braucht und (e) den Grund für Musikgeschichtsschreibung, der zumindest nicht länger - wie in den vergangenen zwei J ahrhunderten - evolutionistisch zu sein braucht.

\section{Gedanken zur Neujustierung von Musikgeschichtsschreibung}

Die Ausführungen zur Geschichte der Musikgeschichtsschreibung hatten zum Ziel, deutlich zu machen, dass musikbezogene Historiographie im Zeitalter der medialen Überlieferung von Musik einer Neujustierung bedarf. Dies ist bislang noch nicht hinlänglich reflektiert geschweige denn umgesetzt worden. Um die notwendige Neujustierung zu ermöglichen, gilt es Fragen wie die folgenden zu klären: 
(1) Benötigen wir im Zeitalter der medialen Überlieferung überhaupt noch Musikgeschichte? Schließlich ist doch praktisch jede auf Tonträger festgehaltene Musik in Archiven gespeichert und zur Nutzung bzw. Analyse verfügbar?

(2) Ist das, was erklingt, wirklich schon die ganze Musik? Gehört nicht auch alles das dazu, was die musikalische Produktionshandlung, die Umstände und die Situation der Darbietung, den Akt des Hörens und den kommerziellen Verwertungszusammenhang betrifft?

(3) Wie vollständig und umfassend sollte Musikgeschichte betrieben werden? Geht es um eine Universalgeschichte der Musik, oder ist eine Auswahl aus den musikalischen Datenmengen sinnvoll, und wenn ja, welche?

(4) Gibt es zwingende Ordnungskriterien oder >historische Narrative<, die einer Musikgeschichte zugrunde gelegt werden sollten? Wenn ja, sind sie nur musikimmanent oder auch kultur- und sozialgeschichtlich, quantitativ bzw. qualitativ, nach Gefallens- vs. Missfallensurteilen u.a.m. auszurichten?

(5) Darf Qualität ein Kriterium sein? Und wenn ja, wer bestimmt, was musikalische Qualität ist?

(6) Für wen ist Musikgeschichtsschreibung heutzutage gedacht? Für die Experten vom Fach, für Verleger und Veranstalter, für die kreativ Schaffenden, für das breite Publikum oder eine spezialisierte Fangemeinde?

Und schließlich

(7) Verbirgt sich hinter Musikgeschichtsschreibung die Hoffnung, Gegenwart beherrschbar zu machen, indem man das Vergangene nach überschaubaren Kriterien strukturiert und dann diese Kriterien als ordnende und sinnstiftende Faktoren der Gegenwart zugrunde legt?

Im Folgenden möchte ich versuchen, einige durchaus persönliche Antworten auf dieses Fragenbündel zu geben, um damit die erforderliche Neujustierung von Musikgeschichtsschreibung voranzutreiben.

1: Ging es bislang in der Musikwissenschaft darum, die in Schriftform vorliegende Musik vergangener Zeiten zu rekonstruieren bzw. quellenkritisch aufzuarbeiten, einer Komponistenpersönlichkeit zuzuordnen, zu analysieren und für die Gegenwart nutzbar zu machen, so drohen wir heutzutage angesichts der Überfülle an medial gespeicherter, in Archiven der unterschiedlichsten Provenienz und im Internet aufbewahrter Musik die Orientierung zu verlieren.

Entsprechend stärker wird der Wunsch nach einer Reduktion der komplexen musikalischen Wirklichkeit. Menschliche Wahrnehmung be- 
ruht auf Auswahl, Distinktion und Schwerpunktsetzung. Gerade weil die Musik aller Zeiten, Stile und Gattungen heutzutage zum beliebigen Gebrauch zur Verfügung steht, bedarf es des ordnenden Eingriffs. Allein durch eine Sichtung des musikalischen Materials nach Kriterien der Verdichtung lässt sich Überschaubarkeit herstellen. Sind die zugrunde gelegten Kriterien wissenschaftlich abgesichert, d.h. exakt, plausibel und nachvollziehbar definiert, so bildet das die Voraussetzung und Grundlage für eine aussagekräftige und bis an die Gegenwart heranreichende Musikgeschichtsschreibung. Die brauchen wir heutzutage nötiger denn je - vor allem, um den Anteil an Vergangenheitsgebundenheit in der neuen Musik zu erkennen, mithin z.B. die retrospektiv-geschichtsträchtigen Bestandteile aktueller Musikproduktionen zu erfassen.

2: Der musikwissenschaftliche Umgang mit notierter bzw. medial gespeicherter Musik macht schnell vergessen, dass Musik aus mehr besteht als dem festgeschriebenen Produkt mit seinen musikalischen Strukturen. Musik ist multidimensional. Die musikalische Produktionshandlung, das musikalische Produkt, die verschiedenen Vermittlungsschritte bis hin zur klanglichen Realisation, ihre Rezeption und schließlich die Vermarktung von Musik bilden eine Einheit: das Beziehungssystem Musik (Rösing 2004: 160ff.). Hinzu kommt die visuelle Ebene, die jede Live-Darbietung begleitet. Sie ging mit der Notation von Musik verloren, ist aber in Musikvideo und Musikclip wieder zu ihrem Recht gekommen ist.

Musikgeschichtsschreibung von heute muss sich der Aufgabe stellen, die Multidimensionalität von Musik mit einem der Komplexität der Sachverhalte gerecht werdenden Forschungsinstrumentarium zu hinterfragen und in ihren Ausführungen zu berücksichtigen. Die Funktionszusammenhänge von Musik als Bedingungssystem angemessen zu gliedern und zu beschreiben ist kein leichtes Unterfangen. Der kreative Schaffensakt, die typischen Darbietungsformen - seien sie live oder medial - und insbesondere das Hörverhalten mit seinen verschiedensten Verhaltensweisen und Ritualen gehören dazu, kurz, die musikbezogenen Verhaltensweisen und Handlungen all jener, die aufgrund ihres Denkens, Fühlens, Wissens und ihrer sozialen Einbindung das Musikleben geprägt haben bzw. prägen. ${ }^{1}$

3: Musikgeschichtsschreibung kann es sich nicht leisten, bestimmte Stile, Gattungen, Richtungen nach der Maßgabe auszuklammern, dass sie nicht geschichtswürdig seien. Die Aufspaltung des Faches Musikwissenschaft in

1 Vgl. Gerhard Schulzes Analysen von alltagsästhetischen Milieus und Schemata des Musikhörens; Schulze 1992: 142ff. u. 630ff. 
historische und vergleichend-systematische Musikwissenschaft, in Musikethnologie, musikalische Akustik, Musikpsychologie, Musiksoziologie, Popularmusikforschung und Musikpädagogik hat mit dazu beigetragen, die traditionelle Wertehierarchie aus der Kunstmusikperspektive als fragwürdig zu dechiffrieren.

Die Aufteilung in die verschiedenen musikwissenschaftlichen Subdisziplinen bietet den Vorteil, dass der Anspruch wissenschaftlichen Arbeitens angesichts der Vielfalt der zur Verfügung stehenden Untersuchungsmethoden zumindest einigermaßen eingelöst werden kann. Einigermaßen deswegen, weil die dem naturwissenschaftlichen Verständnis zu Grunde liegenden Gütekriterien (Objektivität, Wiederholbarkeit, Überprüfbarkeit und Diskursivität) in den Bereichen der Geistes- und Kulturwissenschaften nur bedingt umsetzbar sind. Denn weder fügt sich der Untersuchungsgegenstand Musik in das Raster der musikwissenschaftlichen Teilfächer, noch lassen sich kulturelle Phänomene im Sinn der Naturwissenschaften bündig operationalisieren, ohne dabei Gefahr zu laufen, den Gegenstand zu verlieren.

4: Wie schon angedeutet, gibt es keine zwingenden Auswahlkriterien für die Musikgeschichtsschreibung. Festzuhalten bleibt jedoch, dass sich der Musikbegriff gegenüber früher erheblich geöffnet hat. Gemäß dem Konzept der Oral History (Ong 1982) sollte Geschichtsschreibung sich nicht nur an den Leistungen der Sieger und der Hochkultur orientieren, sondern ebenso die Handlungen der Verlierer und die Gegebenheiten der Alltagskultur berücksichtigen. Bezogen auf Musik bedeutet das: Alle musikalischen Aktivitäten, alle Musik- und Musiziersphären sind zu berücksichtigen. Und dies hat wertneutral zu geschehen. Gerade Wertneutralität aber fällt schwer, war doch die der Hochkultur vorbehaltene Musikgeschichtsschreibung bis in das 20. Jahrhundert hinein vorrangig Bewertungsgeschichte.

Damit soll nun allerdings nicht gesagt sein, dass jetzt dem Fetisch der Vollständigkeit gehuldigt werden muss. Es gibt ja, über die Zeitschiene der Chronologie hinaus, durchaus Ordnungskriterien, die eine Verdichtung erlauben, ohne die komplexe musikalische Wirklichkeit unangemessen zu verfälschen. Historische Narrative wie die Gruppierung nach Stilen, Epochen, Gattungen oder Genres, nach Ensembles und Besetzungen, Bands, Solisten oder Musiziersphären, nach sozialgeschichtlichen, ökonomischen oder musikimmanenten Aspekten können immer dann durchaus angemessen sein, wenn die Gründe für die Auswahl detailliert dargelegt werden. Denn Reduktion ermöglicht Prägnanz. 
Sie ist legitim, solange sie einem Musikkonzept gerecht wird, das - sei es so subjektiv wie auch immer - wahrnehmungspsychologisch ausgerichtet ist und damit den Rahmenbedingungen entspricht, die für alle die gesellschaftlich relevanten Gruppen gilt, die Musik machen oder einen wie auch immer gearteten Umgang mit Musik haben.

5: Gerne wird musikalische Qualität zum Auswahlkriterium erhoben. An die Stelle einer exemplarischen Beschreibung der verschiedenen musikalischen Erscheinungsformen, die das Musikleben bestimmen, erfolgt eine Auswahl nach Gesichtspunkten wie hohe vs. niedere, triviale vs. gehobene, innovative vs. traditionelle, Wahrheit suchende vs. falsches Bewusstsein abbildende Musik (Adorno 1962). Wie sehr aber schon allein im Bereich der gehobenen Musik die Qualitätskriterien je nach Epoche, Zeitgeist und gängigen Modetrends variieren, hat Werner Braun in seinem Buch Musikkritik (1972) überzeugend dargelegt. Zeitenüberdauernde Definitionen von dem, was musikalische Qualität ausmacht, gibt es demnach nicht. Die Kriterien stellen ein Ergebnis des Diskurses über Musik von Kennern und Experten dar. Sie sind zwar historisch bedeutsam, können aber keine Allgemeingültigkeit beanspruchen.

Das desto weniger, wenn es darum geht, die Umgangs- und Gebrauchsmusik bzw. - seit dem ausgehenden 19. J ahrhundert - volkstümliche Musik, Blues, Jazz, Schlager, Pop und Rock in die Geschichtsschreibung mit einfließen zu lassen. Qualitätsmerkmale als Auswahlkriterium sind folglich - wenn überhaupt - für die unterschiedlichen Musiksphären und Zeitabschnitte immer wieder von neuem zu definieren. Dabei muss die Funktion der Musik im Mittelpunkt der Betrachtung stehen. Tanzmusik, Hausmusik, Musik für die Kirche, Militärmusik, Konzertmusik, Arbeits- oder Kinderlieder usw. unterliegen jeweils durchaus eigenen Qualitätsansprüchen: Musikalische Struktur und intendierte Funktion sind hier jeweils genregerecht in Deckung zu bringen.

6: Allzu gerne wird Musikgeschichte als Exkurs von Experten für Experten verstanden. Geringe Auflagezahlen signalisieren, dass ein Großteil der musikgeschichtlichen Veröffentlichungen im inneruniversitären Kreis verbleibt und im besten Fall noch die Musikredaktionen von Zeitung, Rundfunk und Fernsehen erreicht. Das ist bedauerlich. Denn alle, die gerne Musik hören oder machen, die Liebhaber, das breite Publikum, die Fangemeinde haben Anspruch auf ein seriöses, fachlich fundiertes Musikschrifttum. Zwar bedarf Musik keines wissenschaftlichen Kommentars, um verstanden zu werden. Sie ist »in sich selbst genügsam« (Rösing/ Petersen 2000: 16). Wohl aber besteht der Wunsch, sich selbst in 
historisch gewachsenen und gesellschaftlich vermittelten musikalischen Zusammenhängen zu positionieren, die über das persönliche Erfahrungsinventar hinausweisen. Um hier Anregungen zu geben, die über den inneren Zirkel der Expertengruppen hinaus Wirksamkeit entfalten, bietet sich das Internet ein geeignetes Publikationsforum an. Die Koordination und Zusammenschau des Expertendiskurses könnte zu einer Art Wikipedia der Musikgeschichte werden: allgemeinverständlich formuliert, übersichtlich gegliedert und jederzeit erweiterbar bzw. revidierbar.

7: Die musikgeschichtliche Aufarbeitung von Musik der Vergangenheit als Legitimationsraster zur Beurteilung der gegenwärtigen Musikproduktion greift nicht nur zu kurz, sie ist auch problematisch. Die evolutionistisch ausgerichtete musikgeschichtliche Sicht des 19. und beginnenden 20. J ahrhunderts hat - neben vielen anderen Faktoren - mit dazu beigetragen, dass Avantgardemusik und zeitgenössische Musik in zunehmendem Ausmaß ihr Publikum verloren haben. Ihre hochkomplexen und Wiederholung negierenden Strukturen verschließen sich der Mehrzahl der Hörer. Nur wenige Experten schätzen diese Form der intellektuellen Herausforderung. Primär aber ist Musik ein nachhaltig emotional geprägtes Kommunikationsmedium. Ihre emotionale, imaginative und assoziative Kraft entfaltet sie, wenn ihre Machart nicht allzu komplex und das wahrnehmungspsychologische so entscheidende Prinzip der Wiederholung hinreichend gegeben ist.

Wie schon gesagt, sollte das Ziel von Musikgeschichtsschreibung eine wertneutrale Bestandsaufnahme aller musikalischen Erscheinungs- und Umgangsformen beinhalten, die innerhalb des zu behandelnden Zeitabschnitts parallel auftreten. Wir wissen ohnehin nicht, wann aus einer aktuellen Musikhandlung Geschichte wird. Die Teilnehmer des Woodstock-Festivals z.B. hatten anderes im Sinn als an Musikgeschichte zu denken. Erst aus der zeitlichen Distanz heraus wird die historische Bedeutung sichtbar und kann für eine »gliedernde Strukturbeschreibung « des »Wesentlichen « und »Unwesentlichen« herangezogen werden (Dahlhaus 1985: 24).

Obwohl unser Leben von Verwissenschaftlichung und Expertenprognosen immer mehr beeinflusst wird, hat sich gezeigt, dass der Tunnelblick von Experten zu keiner besseren Trefferquote im Hinblick auf Zukunftsprognosen führt als diesbezügliche Statements von Affen (Horx 2011). Mit anderen Worten: Eine Verwissenschaftlichung des Blicks auf die Musik von gestern und heute hilft im Hinblick auf Musikgeschichtsschreibung nur bedingt weiter. Wenn an die Stelle des spontanen Hörens 
die durch Sachkenntnis geprägte Rezeption tritt, so muss das noch lange nicht, wie Dahlhaus und Eggebrecht (1985: 100) postulierten, für den verstehenden Umgang mit Musik unumgänglich sein. Die Durchschaubarkeit von Gegenwart durch ihre Verortung in der Vergangenheit kann allenfalls ansatzweise funktionieren. Denn kulturelle Dynamik unterliegt keinen naturwissenschaftlichen Gesetzen. Die geschichtliche Rekonstruktion von musikalischer Vergangenheit erlaubt nicht unbedingt die Konstruktion des musikalisch Zukünftigen.

\section{Ausblick}

Eine Musikgeschichtsschreibung, die wie in früheren Zeiten ihre Aufmerksamkeit nahezu ausschließlich auf das Werk und die Biographie von Komponistenpersönlichkeiten der Hochkultur richtet, kann dem Anspruch einer wissenschaftlich ausgerichteten Musikgeschichte nicht gerecht werden. Die Ergebnisse aller Teildisziplinen müssen, wie es ja schon Guido Adler 1885 gefordert hat (ohne dass diese Forderung bis heute eingelöst worden wäre!), in die Arbeit mit einbezogen und nicht als »außermusikalisch « abgewertet werden. Seriöse Musikgeschichtsschreibung lässt sich dann allerdings nicht mehr im Alleingang leisten. Sie verlangt nach Teamarbeit. Erst die einzelnen musik- und kulturwissenschaftlichen Teildisziplinen mit ihren unterschiedlichen methodischen Forschungsansätzen ergeben ein neues Ganzes, nämlich eine kulturgeschichtlich, sozialwissenschaftlich und wahrnehmungspsychologisch verortete Musikwissenschaft. Damit wird Musikgeschichtsschreibung zu einer dem komplexen Beziehungssystem Musik angemessenen Konstruktionshandlung. Vorurteilsfreie Sichtung der Quellen, wertneutrale Auswahl nach dem Kriterium der Typik und exemplarische Beschreibung erhöhen den Grad an Objektivität, ohne ein durchaus legitimes persönliches Erkenntnisinteresse und den jeweiligen Gegenwartsbezug von Geschichtsforschung damit zu unterbinden.

Angesichts dieser umfassenden Forderungen an Musikgeschichtsschreibung ist zu klären, wie vollständig sie in Bezug auf die verschiedenen Epochen und Stile zu sein hat. Kann der Traum einer Universal- oder Weltgeschichte der Musik mehr sein als eine Utopie? Ist das überhaupt noch ein Anliegen, nachdem der evolutionäre Traum vom »wilden Geheul « der »Südsee-Insulaner « (Hanslick 1854, zit. n. 1966: 144) zu den hoch entwickelten Werken einer autonomen Musik ausgeträumt ist? Der Gedanke einer musikalischen Weltgeschichte als »Menschheitsgeschichte« bleibt jedoch nach wie 
vor virulent: Kein geringerer als Georg Knepler (1997: 1312) hat das noch zum Ende des 20. J ahrhunderts mit Nachdruck eingefordert.

\section{Literatur}

Adler, Guido (1885). »Umfang, Methode und Ziel der Musikwissenschaft. « In: Vierteljahresschrift für Musikwissenschaft 1, S. 5-20.

Adorno, Theodor W. (1962). Einleitung in die Musiksoziologie. 12 theoretische Vorlesungen. Frankfurt/ M.: Suhrkamp.

Braun, Werner (1972). Musikkritik. Versuch einer historisch-systematischen Standortbestimmung. Köln: Gerig.

Burney, Charles (1776-1789). A General History of Music, from the Earliest Ages to the Present Period. 4 Bde. London: Harcourt, Brace \& Company.

Dahlhaus, Carl (1985). Die Musik des 18. Jahrhunderts (= Neues Handbuch der Musikwissenschaft 5). Laaber: Laaber.

Dahlhaus, Carl / Eggebrecht, Hans Heinrich (1985). Was ist Musik? Wilhelmshaven: Heinrichshofen.

Diderot, Denis / Alembert, J ean Baptiste le Rond d' (Hg.) (1751-1780). Encyclopédie, ou dictionnaire raisonné des sciences, des arts et de métiers. Paris: Briasson, David, Le Breton \& Durand.

Elste, Martin / Schüller, Dietrich (1998). »Tonträger und Tondokumente. «In: Die Musik in Geschichte und Gegenwart. Sachteil, Bd. 9. Hg. v. Ludwig Finscher. Kassel, Stuttgart: Bärenreiter/ Metzler (2. Aufl.), Sp. 646-678.

Fechner, Gustav Theodor (1860). Elemente der Psychophysik. 2 Bde. Leipzig: Breitkopf \& Härtel.

Forkel, J ohann Nikolaus (1788/1801). Allgemeine Geschichte der Musik. 2 Bde. Leipzig: Schwickert.

Graf, Walter (1966). »Zur Verwendung von Geräuschen in der außereuropäischen Musik. « In: Jahrbuch für musikalische Volks- und Völkerkunde 2, S. 59-90.

Hanslick, Eduard (1966). Vom Musikalisch-Schönen. Ein Beitrag zur Revision der Ästhetik der Tonkunst. Wiesbaden: Breitkopf \& Härtel (16. Aufl.; Erstausgabe Leipzig: Rudolf Weigel 1854).

Helmholtz, Herrmann v. (1863). Die Lehre von den Tonempfindungen als physiologische Grundlage für die Theorie der Musik. Braunschweig: Vieweg.

Horx, Matthias (2011). «Wir Krisenverweigerer. «In: Berliner Zeitung vom 15. Dezember, S. 4.

Kaden, Christian (1985). Musiksoziologie. Wilhelmshaven: Heinrichshofen.

Kiesewetter, Raphael Georg (1846). Geschichte der europäisch-abendländischen oder unserer heutigen Musik. Darstellung ihres Ursprungs und ihrer stufenweisen Entwicklung von dem 1. J ahrhundert des Christentums bis auf unsere Zeit. Leipzig: Breitkopf \& Härtel (2. Aufl.).

Knepler, Georg (1997). »Musikgeschichtsschreibung. «In: Die Musik in Geschichte und Gegenwart. Sachteil, Bd. 6. Hg. v. Ludwig Finscher. Kassel, Stuttgart: Bärenreiter/ Metzler (2. Aufl.), Sp. 1307-1319.

Martini, Giovanni Battista (1757-1781). Storia delle musica. 3 Bde. Bologna: Stamperia di Lelio dalla Volpe.

Mattheson, Johann (1722-1725). Critica musica. Hamburg: Selbstverlag / Thomas von Wierings Erben. 
Ong, Walter J. (1982). Orality and Literacy: The Technologizing of the World. New York: Methuen.

Pendzich, Marc (2004). Von der Coverversion zum Hit-Recycling. Historische, ökonomische und rechtliche Aspekte eines zentralen Phänomens der Pop- und Rockmusik. Münster: Lit.

Riemann, Hugo (1901). Geschichte der Musik seit Beethoven (1800-1900). Berlin, Stuttgart: Spemann.

Rösing, Helmut (2004). »9/11. Wie politisch kann Musik sein?« In: 9/11 - The world's all out of tune. Populäre Musik nach dem 11. September 2001. Hg. v. Dietrich Helms und Thomas Phleps (= Beiträge zur Popularmusikforschung 32). Bielefeld: transcript, S. 155-168.

Rösing, Helmut / Petersen, Peter (2000). Musikwissenschaft. Was sie kann, was sie will. Reinbek: Rowohlt.

Rousseau, J ean-J acques (1768). Dictionnaire de musique. Paris: La Veuve Duchesne Libraire.

Schulze, Gerhard (1992). Die Erlebnisgesellschaft. Kultursoziologie der Gegenwart. Frankfurt/ M.: Campus.

\begin{abstract}
In recent years the conditions for writing about the history of music evidently have changed. Today we can realize what in 1885 was explained by Guido Adler in his programmatic sketches concerning a new musicology. Large digital databases worldwide store the notated as well as not-notated music of past and presence. This is the sonic material for the writing of a comprehensive and genre-overlapping music history. Therefore music history can no longer be a question of value - as in the evolutionistic drafts of the $19^{\text {th }}$ and early $20^{\text {th }}$ centuries. More important seems to be the description of music in terms of its multidimensional conditions: from its creation as a performance up to its reception and merchandizing. This requires a methodological pluralism and the cooperation of historical, systematic-comparative, acoustic and psychological, pedagogic, social and economic views.
\end{abstract}

